\title{
DE LA LECTURA INTENSIVA A LA EXTENSIVA EN LA CLASE DE ILE. FUNDAMENTOS DIDÁCTICOS ${ }^{1}$
}

\author{
Raúl Ruiz Cecilia, Universidad de Granada \\ Email: raulruiz@ugr.es
}

\begin{abstract}
Resumen: El presente artículo aborda la enseñanza de la lectura en el aula de Inglés como Lengua Extranjera (ILE). Numerosos investigadores han resaltado los beneficios que la lectura proyecta en el lector y cómo texto y sujeto interactúan para ampliar los límites impuestos por el formato físico. Ahora bien, pocos son los que han abogado por un desarrollo del goce estético en la lectura que se lleva cabo en el aula de ILE. Se han priorizado enfoques reduccionistas centrados en los aspectos formales del lenguaje dejando de lado aproximaciones más globales y relacionadas directamente con el sujeto que lee. En este trabajo se hace una revisión de los principios que rigen la lectura intensiva y extensiva y cómo se podrían utilizar para enseñar inglés a no nativos.

Palabras clave: Lectura intensiva, lectura extensiva, inglés como lengua extranjera,
\end{abstract} enseñanza

Title in English: From Intensive to Extensive Reading within the EFL Class. Teaching implications

Abstract. This article tackles the teaching of reading within the area of English as a Foreign Language (EFL). Several researchers have pointed out the benefits that reading projects on the reader and how texts and readers interact in order to go beyond format constraints. However, not many have advocated for a second language reading approach where aesthetic enjoyment occupies a central role. Reductionist ideas focused on formal aspects of language have been prioritized over other global approaches centered on the reader. This paper reviews the bases of intensive and extensive reading and how they could be used to teach English to non-native speakers.

Keywords: Intensive reading, extensive reading, English as a Foreign Language, teaching

\section{INTRODUCCIÓN}

El recuerdo que deja un libro es más importante que el libro mismo.

Gustavo Adolfo Bécquer

La enseñanza de lenguas extranjeras ha sido objeto de estudio en las distintas corrientes lingüísticas que se han sucedido en las últimas décadas, desde el estructuralismo de

Date of reception: 5 May 2011

Date of acceptance: 30 Nov 2011 
Saussure, el generativismo iniciado con Chomsky, hasta el funcionalismo que aglutina los paradigmas teóricos de la Escuela de Praga, la glosemática de Louis Hjemslev, los trabajos de André Martinet, la gramática sistémica de Michael Halliday y la gramática funcional de Simon Dik.

Todos estos movimientos lingüísticos han considerado el desarrollo de las destrezas básicas como pilar fundamental para el dominio de la lengua extranjera (aunque los índices de priorización o relevancia difieren de un planteamiento a otro). En el artículo que nos ocupa, nos centraremos en la lectura y cómo se desarrolla en los enfoques actuales de enseñanza de lenguas extranjeras, principalmente el método comunicativo (basado en el funcionalismo). El objetivo fundamental es hacer una revisión crítica de los tipos de lectura que los profesionales de la educación utilizan a la hora de enseñar inglés como lengua extranjera.

\section{CONSIDERACIONES PREVIAS}

En 1978 PUGH y posteriormente en 1979 LUNZER y GARDNER describieron varios estilos de lectura. Su estudio transcendió el ámbito para el que fue creado y aportó nuevos términos específicos al campo de la didáctica de las lenguas extranjeras. Los tipos de lectura a los que hacemos referencia los recoge TRICIA HEdGE en su libro Teaching and Learning in the Language Classroom (2002: 195). Son:

- Lectura receptiva: Se da cuando la persona lectora quiere disfrutar del material impreso o simplemente quiere entender lo que se describe en un artículo...

- Lectura reflexiva: Se lee un pasaje y después interrumpimos el proceso de lectura para reflexionar o contrastar lo que acabamos de leer.

- Lectura global (skimming). En este caso el objetivo es conseguir una impresión global del contenido del texto.

- Lectura selectiva (scanning). Si el propósito del lector es localizar una información específica, entonces nos encontraremos ante un caso de scanning.

- Lectura intensiva o analítica: El lector realiza un estudio exhaustivo y detallado del texto.

Como consecuencia, estos diferentes estilos de lectura derivarán en la activación de múltiples y variadas estrategias para satisfacer las intenciones que cada lector se plantee.

Esta clasificación, desde nuestro punto de vista, puede reformularse y simplificarse en la siguiente figura:

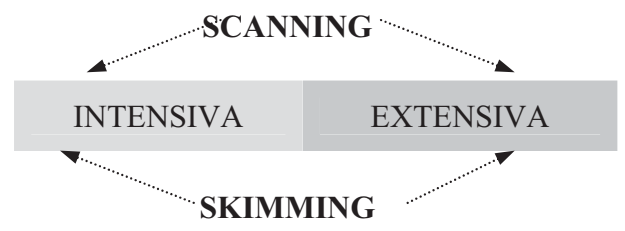

Fig 1. Interrelaciones entre estrategias y tipos de lectura. 
Los dos tipos de lecturas fundamentales en el aula de idiomas son, de acuerdo con la figura anterior, la lectura intensiva y la extensiva. El scanning (búsqueda de información específica) y el skimming (perseguir la idea global del texto) se convierten en estrategias a la que pueden recurrir lectores en su proceso individualizado de lectura (ya realicen un tipo u otro de lectura).

El siguiente cuadro (adaptado de WELCH 1997) muestra esquemáticamente las relaciones entre lectura extensiva y lectura intensiva:

\begin{tabular}{|l|c|l|}
\hline \multicolumn{1}{|c|}{ Extensiva } & \multicolumn{1}{|c|}{ Lectura } & \multicolumn{1}{c|}{ Intensiva } \\
\hline $\begin{array}{l}\text { Comprensión general y lectura por } \\
\text { placer }\end{array}$ & OBJETIVO & Estudio exhaustivo del lenguaje \\
\hline Fácil & NIVEL & Más complicado \\
\hline Mucha & CANTIDAD & Escasa \\
\hline Rápido y con fluidez & VELOCIDAD & Lento \\
\hline
\end{tabular}

Fig 2. Comparación entre lectura intensiva y extensiva.

\section{LA LECTURA INTENSIVA EN LENGUA EXTRANJERA}

La lectura intensiva ha sido una práctica habitual (y lo sigue siendo) en las aulas de idioma extranjero y una de las más perpetuadas en los libros de textos publicados para la enseñanza/aprendizaje del inglés como lengua extranjera. Normalmente el texto se orienta hacia el estudio de aspectos lingüísticos (gramaticales, léxicos, cohesivos o pragmáticos), hacia la comprensión total del contenido textual, o bien, según BAMFORD y DAY (1997), hacia la persecución de una cuidadosa práctica íntimamente relacionada con la enseñanza de los componentes de la lectura.

El Texas Center for the Advancement of Literacy \& Learning fundamenta la lectura intensiva en la comprensión detallada de la información del texto. Para ello cita siete principios fundamentales:

1) Visión de conjunto: ésta se puede lograr por medio de varias estrategias lectoras como son el skimming (apuntar hacia la idea global del texto); el aprovechamiento de la información derivada del título; la lectura de las primeras líneas del texto; la observación de ilustraciones, subtítulos, notas a pie de página... Esta visión contribuye a una mejor organización posterior de la información textual.

2) Propósito: el sujeto lector predetermina el objetivo que quiere conseguir con la lectura enfocando, de este modo, sus estructuras mentales hacia la consecución de tal fin. Siguiendo los principios teóricos del Texas Center for the Advancement of Literacy \& Learning, el lector se debe plantear:

- ¿Qué información necesitamos?

- ¿Qué nivel de detalle se necesita con respecto a la información?

- ¿ ¿Dónde se pone el énfasis: en las ideas, secuencias, hechos concretos...?

- ¿Durante cuánto tiempo tenemos que retener la información: hasta la evaluación, durante un período más largo, toda la vida...? 
- ¿Cómo utilizamos la información: para pensar, para redactar un informe, para hacer un examen?

1) Preguntas: éstas tienen que seguir el mismo orden que presenta la información del texto.

2) Lectura: se trata de una lectura línea a línea guiada por nuestro objetivo y las preguntas que tengamos que contestar.

3) Resumen: por medio del resumen se persigue la reorganización de las ideas principales y la de las ideas secundarias. Se propone ir párrafo a párrafo para no olvidar ninguno de los puntos esenciales.

4) Evaluación: se trata de una autoevaluación de la información retenida durante el proceso de lectura intensiva. Se prepara así al docente para superar con éxito el examen implementado posteriormente.

\section{5) Comprensión}

\section{LA LECTURA EXTENSIVA EN LENGUA EXTRANJERA}

[...] the only explanation of why extensive reading is effective is that it replicates the process by which we learn to read in our native language, that is, "prolonged practice". (NeLL 1988: 84).

En el terreno de la enseñanza de la lectura en lengua extranjera, las últimas tendencias apuntan hacia la utilización de la lectura extensiva como motor para desarrollar la animación y el gusto por la lectura y como fuente para mejorar la literacy del estudiantado (entre otros, Krashen 1993; Elley 1991; Mason y Krashen 1997; Maley 1999; Kern 2000; Day y BAMFORD 2000, 2002; BROWN 2001)

Una práctica bastante generalizada en el aula de idiomas es ver el texto como un objeto destinado sólo y exclusivamente a su estudio lingüístico dejando de lado su valor estético. La práctica de la lectura extensiva se presenta como una alternativa para despertar la motivación del alumnado e involucrarlo en los avatares estéticos y receptivos de la obra de arte. La lectura extensiva es la clave para el desarrollo de la habilidad lectora, la competencia lingüística, el vocabulario, la ortografía y la escritura.

Los fundamentos teóricos generales coinciden de un estudioso a otro aunque suelen aparecer ciertas diferencias de priorización entre ellos. A continuación se revisarán varios modelos propuestos haciendo especial hincapié en el propuesto por DAY y BAMFORD (2000).

De acuerdo con DAY y BAMFord (2000: 7-8) y DAY y BAMFord (2002: 136-141), las características de un programa de lectura extensiva en lengua extranjera (LE) deben ser:

1) Los estudiantes tienen que leer la mayor cantidad de material posible. Normalmente leen un libro cada semana (dependiendo de las horas de docencia que se destinen a la realización de un programa de lectura extensiva).

2) Tiene que haber disponibilidad de materiales con una temática variada. Es aconsejable la elaboración de una biblioteca de aula que responda a los intereses de los sujetos lectores implicados. 
[...] ideally be as varied as the learners who read them and the purposes for which they want to read [...] Learners are led to read for different reasons (e.g. entertainment; information; passing the time) and, consequently, in different ways (e.g., skimming; scanning; more careful reading). (DAY y BAMFORD, 2002: 137).

3) Los estudiantes seleccionan lo que quieren leer. El docente determina solamente la cantidad de lecturas a la semana/mes/trimestre. La elección será libre y se les animará a interrumpir la lectura si no les aporta nada.

If the student finds the book too difficult to enjoy, the extensive reading procedure requires that the book be changed, and not the student be made to study it more closely. The level of global understanding required varies with the student's language proficiency, the nature of the text, and other factors. (SusSER y Roвb, 1990).

Tenemos que crear una atmósfera que propicie y haga que los estudiantes tomen esta iniciativa puesto que normalmente el sistema educativo los hace totalmente dependientes de los intereses del profesorado. Samuels (1991: 17) comenta al respecto: "[...] unless we phase out the teacher and phase in the learner, many of our students will fail to become independent because throughout their education they were always placed in a dependent role - dependent on the teacher."

4) Los objetivos de la lectura se relacionan con el placer de leer, conseguir información o enfocarse en la comprensión general. La libertad para la lectura que se menciona en el punto tres hace que el aprendiz marque sus intereses en la lectura.

5) La lectura es un premio en sí misma. Es decir, ésta no debería impregnarse de un carácter de obligatoriedad ni orientarse hacia fines evaluadores. El niño o la niña tienen que aprender a ver la actividad lectora como una recompensa en sí misma, un acto personal en el que entrarán en juego las vivencias e intereses del sujeto lector. Si la lectura se focaliza hacia la evaluación, probablemente el aprendiz no disfrute del proceso y desarrolle una actitud negativa hacia la misma. Las actividades deberían estar dirigidas hacia el desarrollo integral de la persona a través de una interacción textual.

6) Los materiales de lectura deben encontrarse dentro del margen de competencia lingüística de los estudiantes. Si las lecturas propuestas son demasiado difíciles, los alumnos desistirán. Esto se hace aún más evidente en la clase de lengua extranjera donde el desconocimiento por parte del alumnado de las estructuras gramaticales, matices culturales o las barreras léxicas hacen inaccesible la parte conceptual del texto. Podríamos invertir la formulación de la Input hipótesis $(i+1)$ de Krashen (1989) y afirmar que para que un texto sea idóneo en la clase de ILE, su nivel de complejidad léxico-sintáctica tiene que situarse un poco por debajo de la competencia general del estudiante. Esto es, $i-1$ según la formulación del autor. De esta forma, se evitarán las continuas interrupciones o acumulación de lagunas que sin duda alguna se reflejarán en la interpretación y goce estético del texto.

DAY y BAMFORD (2002: 137) exponen que para que el estudiantado lea con comodidad en los niveles iniciales, no deben existir más de dos palabras desconocidas por página, mientras que para los niveles intermedios es cinco palabras desconocidas por página. 
7) La lectura es un acto individual y se realiza en silencio. Posteriormente, los alumnos pueden hacer una puesta en común relacionada con su experiencia lectora y de esta forma socializarla y colectivizarla.

8) La velocidad lectora suele ser mayor puesto que el objetivo principal no es una comprensión pormenorizada. Al no tener la presión de alcanzar una comprensión del cien por cien del texto, el sujeto lector agiliza el proceso y será él el que decida si ralentizar ciertas partes o acelerar otras. Así DAY y BAMFORD (2002: 138) añaden:

$[\ldots]$ when learners are reading material that is well within their linguistic ability, for personal interest, and for general rather than academic purposes, it is an incentive to reading fluency [...]. Extensive reading is a chance to keep reading, and thus to practice such strategies as guessing at or ignoring unknown words or passages, going for the general meaning, and being comfortable with a certain level of ambiguity.

9) Los docentes actúan como orientadores para que se consigan los objetivos del programa, explican la metodología, hacen un seguimiento de las lecturas de los estudiantes y guían a los mismos para que saquen el mayor provecho posible del programa de lectura.

10) El docente es tomado como lector modelo por los estudiantes. Es importante que vean explícitamente los hábitos lectores del docente ya que éste es tomado como un ejemplo a seguir.

BELL (1998), por otro lado, en su programa de lectura extensiva prima y añade otras características, algunas de las cuáles se dejan intuir en la taxonomía de DAY y BAMFORD. La idea subyacente de lectura como placer y de protagonismo del sujeto lector, sigue latente en el planteamiento de Bell. A continuación analizaremos los principios que éste considera.

El primero hace referencia al traspaso de poderes en dirección al lector. El aprendiz es el único protagonista de la lectura y debe involucrarse en todo lo que rodee al proceso de lectura (elección de material, tiempo que dedica al proceso lector...). DAY y BAMFORD (2000 y 2002) no lo explicitan directamente pero sí lo dejan entrever en los criterios 3 y 9.

En el segundo de los puntos propone entrevistas periódicas entre el docente y el discente puesto que inducen a la motivación. Demostrando la entrega en su propia lectura, el profesorado puede manifestar actitudes positivas hacia ésta. En la taxonomía anterior, los autores lo incluyen dentro del criterio 9, donde el docente actúa de guía y hace un seguimiento abierto y dialógico con el sujeto lector.

La lectura en voz alta es un criterio que no tienen en cuenta DAY y BAMFORD. Para éstos últimos la lectura debe ser siempre un acto individual y en silencio. Bell, por su lado, considera importante la introducción de la lectura en voz alta en la praxis del aula ya que cantidades mesuradas pueden beneficiar la atención del discente y motivarlo hacia el acceso independiente a nuevos textos.

El siguiente criterio de Bell lo constituyen exposiciones por parte de los estudiantes ante el grupo social clase. Estás prácticas pueden ser un buen ejercicio para compartir las experiencias vividas durante el proceso de lectura y hacer copartícipe al resto de compañeros. La opinión de sus iguales sobre un libro puede ser más influyente que la que puedan recabar de otras fuentes. 
La unión de escritura y lectura (literacy) es la propuesta que este investigador hace en el quinto criterio. Tal y como hemos expuesto a lo largo del presente artículo, uno de los beneficios de la lectura extensiva se traduce en una mejora de la destreza escrita.

Bell también considera importante introducir material de audio en el programa de lectura extensiva integrando, de esta manera, las dos destrezas receptivas: comprensión escrita y comprensión oral.

Si se quiere mantener el espíritu individualista, creativo y relajado que propugna el enfoque de lectura extensiva, es necesario que se evite el uso de exámenes que emitan un juicio numérico sobre su actuación.

La disuasión de la utilización abusiva del diccionario constituye el octavo principio de Bell. Si los aprendices concentran toda su energía en la mera descodificación, el mensaje pasará a un segundo plano y el deleite susceptible de producirse con la lectura se convertiría en una tarea ardua y desmotivadora.

El docente, igualmente, monitoriza la lectura de los estudiantes. Los profesionales de la educación guían, orientan, sugieren posibles lecturas a sus pupilos y mantienen un ambiente distendido con los mismos.

Finalmente, propugna mantener el carácter de entretenimiento (criterio ligeramente relacionado con los 1, 2, 3 y más directamente con el 4 de DAY y BAMFORD 2000 y 2002). Bell, a diferencia, sugiere el uso de material multimedia para promocionar los libros y utilizar anécdotas que puedan captar la atención de los potenciales sujetos lectores.

\subsection{Beneficios de la lectura extensiva en el aprendizaje de la lengua extranjera}

Recogemos a continuación algunos de los beneficios que diferentes investigadores de lectura extensiva han resaltado en relación con el aprendizaje de la lengua extranjera.

1) Suministra input comprensible. (KRASHEN, 1992).

2) Aumenta la competencia lingüística general del estudiante (gramática, léxico y estructura textual). (Bell 1998; Grabe 1991; Paran 1996; Lituanas, Jacobs y RENANDYA 1999).

3) Aumenta la exposición del estudiante a la lengua objeto. (ElLey, 1991; Bell 1998).

4) Puede mejorar la escritura y la lectura en lengua extranjera. (HAFIz y Tudor 1989; Robb y Susser 1989; Lituanas, Jacobs y Renandya 1999).

5) Les motiva a leer (BELL 1998), (DAY y BAMFORD 2002) y a crear un hábito de lectura (Lituanas, Jacobs y Renandya 1999).

6) Consolida lo anteriormente aprendido (Krashen 1993).

7) Contribuye a fomentar la confianza en sí mismos (Kembo 1993).

8) Anima a los estudiantes a la explotación de elementos repetitivos y/o superfluos del texto (Bell 1998).

9) Facilita el desarrollo de habilidades de predicción (NunAN 1991). 
10) Aumenta el conocimiento del mundo (Lituanas, Jacobs y Renandya 1999).

\subsection{Ejemplos de programas de lectura extensiva llevados a cabo en el terreno de las lenguas extranjeras}

\begin{tabular}{|c|c|}
\hline Llevado a cabo por... & Descripción de la experiencia \\
\hline $\begin{array}{l}\text { Saragi, Nation y Meis- } \\
\text { TER (1978) }\end{array}$ & $\begin{array}{l}\text { El objetivo estribaba en justificar la lectura extensiva como una for- } \\
\text { ma de individualizar la instrucción léxica y analizar cómo los niveles } \\
\text { de vocabulario del grupo control mejoran con respecto al resto de } \\
\text { compañeros. La obra que utilizaron fue A Clockwork Orange. El } \\
\text { estudio se aplicó a } 20 \text { estudiantes de inglés LE que leían la novela } \\
\text { de Burguess por primera vez y, por lo tanto, no tenían conocimiento } \\
\text { de las } 241 \text { palabras nasdat que aparecen en la obra. Los estudian- } \\
\text { tes pensaban que iban a ser evaluados acerca de la comprensión y } \\
\text { no con respecto al vocabulario. Obtuvieron un } 76 \% \text { de correlación } \\
\text { entre la frecuencia de una palabra nasdat determinada y los tests de } \\
\text { respuestas múltiples que aplicaron. }\end{array}$ \\
\hline $\begin{array}{l}\text { ElLEY y MangubHai } \\
\text { (1983) }\end{array}$ & $\begin{array}{l}\text { Se llevó a cabo en un colegio de las islas Fiji. Su objetivo era ver el } \\
\text { efecto que tenía en el desarrollo de las destrezas lectoras de los estu- } \\
\text { diantes la lectura de libros de historias interesantes. Después de dos } \\
\text { años, los resultados obtenidos en este grupo fueron superiores a los } \\
\text { de otro grupo cuyas lecturas estaban más controladas y no atendían } \\
\text { a los intereses de los discentes. }\end{array}$ \\
\hline HAMP-LyONS (1985) & $\begin{array}{l}\text { Describe el desarrollo de un curso de lectura extensiva (desde la } \\
\text { concentración en los macro niveles de lectura a los aspectos más } \\
\text { cognitivos). }\end{array}$ \\
\hline HAFIZ y Tudor (1989) & $\begin{array}{l}\text { Después de implementar un programa de lectura extensiva, el grupo } \\
\text { experimental mejoró sobre todo la destreza de expresión escrita. }\end{array}$ \\
\hline DAVIS (1995) & $\begin{array}{l}\text { El lugar de la investigación fue Singapur. Se seleccionaron cuarenta } \\
\text { estudiantes de secundaria que presentaban problemas en inglés como } \\
\text { lengua extranjera. Durante los cinco años que duró el experimento, } \\
\text { se consiguió que su nivel (en un principio muy básico) superara la } \\
\text { media nacional. }\end{array}$ \\
\hline Mason y KRASHEN (1997) & $\begin{array}{l}\text { Llevaron a cabo tres estudios en tres instituciones educativas japone- } \\
\text { sas de Osaka. Los resultados muestran que los aprendices mejoraron } \\
\text { ampliamente su comprensión y expresión escrita en comparación } \\
\text { con otros sujetos que no participaron en el experimento. }\end{array}$ \\
\hline $\begin{array}{l}\text { LituANAS, JACOBS y } \\
\text { RENANDYA (1999) }\end{array}$ & $\begin{array}{l}\text { La experiencia se llevó a cabo en Mindanao (Filipinas). Su objetivo } \\
\text { estribó en la utilización de la lectura extensiva como importante he- } \\
\text { rramienta para desarrollar las estrategias lectoras de los estudiantes } \\
\text { (en lengua materna L1 y en lengua extranjera L2). }\end{array}$ \\
\hline $\begin{array}{l}\text { IKUMI HitosugI y DAY } \\
\text { (2004) }\end{array}$ & $\begin{array}{l}\text { También se realizó en la Universidad de Hawai con estudiantes de } \\
\text { japonés (nivel inicial). Se utilizó literatura infantil japonesa como } \\
\text { material para la lectura extensiva. Después de tan sólo diez semanas, } \\
\text { los catorce sujetos del estudio vieron su actitud hacia la lectura posi- } \\
\text { tivamente afectada y su información cultural incrementada. }\end{array}$ \\
\hline
\end{tabular}

Fig. 3. Programas de lectura extensiva

Odisea, $n^{\circ} 12$, ISSN 1578-3820, 2011, 217-232 


\subsection{La lectura extensiva y el desarrollo del potencial léxico del sujeto lector}

La lectura extensiva pone el énfasis en el significado del texto y este simple hecho la diferencia de cualquier otro tipo de lectura. No se pretende el desarrollo de aspectos gramaticales ni siquiera la consolidación directa de los mismos (indirectamente, el lector sí se nutre de todos los elementos que conforman el texto. Inconscientemente, se adquiere fluidez en la LE y se amplían todas las competencias lingüísticas). Leer desde la óptica de la lectura extensiva conlleva el leer por placer, por querer ser copartícipe de todo aquello que acaece en otro mundo paralelo el cual se torna familiar y distante en dosis similares.

Extensive reading involves reading with the focus on the meaning of the text. In general extensive reading does not involve much additional language use other than filling out a brief look report form. From a vocabulary perspective, it is useful to distinguish two types of extensive reading: one which aims at vocabulary growth and one which aims at fluency development. (NATION, 2001: 150).

Los beneficios de la lectura extensiva son múltiples y la mayoría de las veces subjetivos pues dependen de los intereses del sujeto lector en cada momento. No obstante, varios estudios (NAtion 2001, HuAng y VAn-Naerssen 1987, Green y Oxford 1995) demuestran que las ventajas lingüísticas son muy numerosas. La lectura extensiva es un acto de libertad de acción para todo sujeto lector ya que serán ellos los que decidan qué leer, cuándo hacerlo y, la mayoría de las veces, dónde hacerlo.

NAtion (2001: 150) arroja alguna luz sobre el porcentaje ideal que los textos destinados a lectura extensiva deberían tener para ser considerados idóneos. Según él, no debería haber más de un $2 \%$ para asegurar la comprensión y la deducción, ni menos de 1-2\% para que se produzca un incremento del vocabulario y de la fluidez del sujeto lector.

Ahora bien, si queremos que el programa de lectura extensiva contribuya, además de a formar individuos sociales, a ampliar el léxico, los estudiantes deberían tener la oportunidad de encontrarse con la misma palabra en varios contextos. El docente podría hacerlo de dos maneras según Nation. La primera sería asegurando que el discente realiza grandes cantidades de lectura extensiva con lo cual se multiplican las posibilidades de volver a enfrentarse a ese término. Otra de las opciones consistiría en un estudio concienzudo y a propósito de la palabra en cuestión fuera de contexto. Es decir, recurrir a otros ejemplos que complementen la adquisición y aprendizaje pero que han sido previamente preparados por el docente.

El conocimiento léxico es una muestra de riqueza lectora, conocimiento del mundo y habilidad lectora. La enseñanza aislada del vocabulario ignora la importancia que cada palabra por sí misma supone para el conocimiento del mundo.

Por otro lado, estudios bien documentados llevados a cabo (MEZynski 1983; STAHL y Clark 1987; NAtion 2001) demuestran que la pre-enseñanza del vocabulario ayuda a la comprensión siempre y cuando se haga de forma comunicativa y significativa.

$[\ldots]$ the well reported and well conducted studies show that preteaching vocabulary helps comprehension if the preteaching involves rich instruction. That is, the preteaching involves several meetings with the word, focuses on many aspects of what is involved 
in knowing a word including fluency of access of the word (...) and meeting the word in several sentence contexts (...), and gets the learners actively involved with processing the word. (NATION, 2001: 157).

\subsection{Biblioteca de aula}

La implementación de un programa de lectura extensiva en la clase de inglés como lengua extranjera necesita de una biblioteca de aula realista, es decir, una biblioteca de aula que responda a los niveles existentes (heterogéneos), intereses, inquietudes, gustos, motivaciones y demandas de los potenciales sujetos lectores. Además tiene que ser un elemento en continuo reciclaje y receptivo a ampliar su oferta según sea la demanda (intraanual o interanual). De este modo, se convierte en un elemento co-creativo que atiende a la diversidad y se adapta a las peculiaridades individuales de cada uno.

Por ello, en la elaboración de la biblioteca de aula que proponemos, es necesaria una fase en la cual se recabe información que responda a la filosofía subyacente del párrafo anterior: libros para los estudiantes. Tal vez, el instrumento de recogida de datos más interesante sea el análisis de necesidades (needs'analysis). En él suelen aparecer preguntas que reconstruyen el historial lector previo $\mathrm{y}$, al mismo tiempo, señalan la futura trayectoria que le interesa. No se debe olvidar el carácter personal e individual del proceso lector, por lo que la heterogeneidad de los datos será una característica inherente a esta recogida de información. A continuación exponemos un análisis de necesidades que creemos arrojará luz sobre el material a incluir.

1. De todas las lecturas que has realizado en lengua extranjera, ¿cuál ha sido la que más te ha gustado?

2. ¿Quién te recomendó el libro?

3. ¿Se trataba de una lectura graduada o de una lectura en versión íntegra?

4. ¿Qué prefieres: lecturas graduadas o lecturas auténticas?

5. ¿Cuál es tu nivel de lectura?

6. ¿Con qué género literario te sientes más identificado?

7. ¿Qué te gustaría leer este año/curso?

8. ¿Cuántos libros te gustaría leer este año/curso?

9. ¿Te gustaría recibir ayuda del profesor para la elección del libro?

10. ¿Cuáles crees que son los efectos beneficiosos de la lectura?

11. ¿Estás familiarizado con el diario de lectura?

Hedge (1987: 53-56) considera otros instrumentos para la recogida de datos. Por un lado diseña un cloze test con el objetivo de conocer el nivel más apropiado para el grupoclase. Por otro lado, estima necesario que el docente se auto-aplique un cuestionario dónde queden reflejados los objetivos planteados por el mismo. 
Con respecto al cloze test, la investigadora propone buscar extractos de textos diferentes y organizarlos por niveles (atendiendo a sus propios criterios). A continuación procedería a hacer una evaluación poco exhaustiva de la competencia lingüística del grupo-clase apoyándose en el corpus seleccionado. Seguidamente, agrupa lecturas que se correspondan con los resultados de nivel obtenidos. Finalmente, prepara el cloze test utilizando varios ejemplos de los extractos anteriores y lo aplica al grupo-clase.

Centrándonos ahora específicamente en la elaboración de dicho test, HedGE (1987: 53-56) lo estructura de la siguiente forma:

1) Elegir tres pasajes de un libro al azar de unas 200-250 palabras.

2) Copiarlos. Dejar dos o tres frases completas al principio para facilitar el contexto. A partir de ese momento, eliminar una palabra cada ocho. El hueco en blanco tiene que ser homogéneo a lo largo de los fragmentos.

3) Los estudiantes tendrán que rellenar los huecos colocando una sola palabra en cada uno de ellos. El número de palabras correctas será indicativo de cómo el alumno es capaz de reconstruir el mensaje del autor sirviéndose del conocimiento estructural y contextual.

4) A continuación, habrá que corregir los tests para posteriormente dar un porcentaje al alumnado que refleje su nivel como sujetos lectores. Los niveles (en relación con los porcentajes) se recogen en la siguiente tabla:

\begin{tabular}{|l|l|l|l|}
\hline \multicolumn{1}{|c|}{$\%$} & \multicolumn{1}{|c|}{ Nivel } & \multicolumn{1}{|c|}{$\begin{array}{c}\text { Importancia para el } \\
\text { sujeto lector }\end{array}$} & \multicolumn{1}{c|}{ Sugerencia } \\
\hline $60 \%$ & Nivel independiente & $\begin{array}{l}\text { Puede trabajar satis- } \\
\text { factoriamente por sí } \\
\text { mismo }\end{array}$ & $\begin{array}{l}\text { Libro adecuado para } \\
\text { lectura extensiva }\end{array}$ \\
\hline $40-60 \%$ & Nivel práctico & $\begin{array}{l}\text { Puede necesitar ayuda } \\
\text { del docente }\end{array}$ & $\begin{array}{l}\text { Mejor un libro más } \\
\text { sencillo }\end{array}$ \\
\hline$-40 \%$ & Nivel bajo & $\begin{array}{l}\text { Necesitará bastante } \\
\text { ayuda por parte del } \\
\text { docente }\end{array}$ & Descartar el libro \\
\hline
\end{tabular}

Fig. 5. Elección de lecturas para el aula (adaptado de Hedge, 1987: 55).

El otro instrumento, el cuestionario centrado en el docente, se orienta hacia la autorreflexión, es decir, el profesor se plantea qué grado de conocimiento o desconocimiento tiene de sus discentes. Asimismo, establece una relación entre los potenciales aprendices y los objetivos preestablecidos para el curso. 
1) ¿Cuáles son las características de mi clase como grupo?

- ¿Cuántos estudiantes hay?

- ¿Qué edad tienen?

- ¿Qué distribución por sexos hay?

2) ¿Cuánto conozco de mis estudiantes como seres individuales?

- ¿Cuáles son sus antecedentes culturales?

- ¿Qué experiencias tienen sobre lectura en LE?

- ¿Cuáles son sus intereses personales con relación a la lectura?

3) ¿Qué sé sobre las habilidades lectoras de mis estudiantes?

- ¿Han hecho algún examen donde queden reflejadas?

- ¿Puede algún otro profesor orientarme?

- ¿Existe algún documento que me aporte más información?

4) ¿Qué información tengo respecto al nivel del lenguaje de los libros?

- ¿Se recoge el número de palabras?

- ¿Explica la editorial la relación entre el nivel del libro y la competencia del estudiante o el libro de texto?

- ¿Hay algún método para hacer una valoración aproximada del libro?

- ¿Se puede utilizar algún test de readability?

5) ¿Son apropiados los libros para los/las estudiantes?

- ¿Conozco la edad recomendada?

- ¿Algún otro docente ha utilizado el libro con anterioridad?

- ¿Interesa el contenido a los estudiantes?

- ¿Cuál es el escenario cultural donde transcurre la acción?

- ¿Hay un glosario?

6) ¿Está bien presentado?

- ¿Hay ilustraciones atractivas y apropiadas?

- ¿Es apropiado el tamaño de letra y el formato de las páginas?

- ¿Contribuye la composición general del libro a la comprensión?

Fig. 6. Cuestionario para elegir lecturas de aula (Adaptado de Hedge, 1987: 61).

Otro campo de interés, resultado de la implementación de algunas de las herramientas anteriores, se orienta hacia las categorías temáticas (géneros literarios) que debemos incluir en la biblioteca. La siguiente tabla puede servir de modelo para el docente una vez éste se enfrenta a la selección de lecturas (Ruiz CECILIA, 2005): 


\begin{tabular}{|c|c|c|c|}
\hline $\begin{array}{c}\text { GÉNERO LITERA- } \\
\text { RIO }\end{array}$ & $\begin{array}{c}\text { TÍTULOS SUGERI- } \\
\text { DOS }\end{array}$ & $\begin{array}{l}\text { TIPO DE LECTURA } \\
\text { (GRADUADA/ } \\
\text { AUTÉNTICA) }\end{array}$ & $\begin{array}{l}\text { NIVEL RECOMEN- } \\
\text { DADO }\end{array}$ \\
\hline \multicolumn{4}{|l|}{ Realista } \\
\hline \multicolumn{4}{|l|}{ Romántico } \\
\hline \multicolumn{4}{|l|}{ Fantástico } \\
\hline \multicolumn{4}{|l|}{ Aventuras } \\
\hline \multicolumn{4}{|l|}{ Ciencia Ficción } \\
\hline \multicolumn{4}{|l|}{ Misterio } \\
\hline \multicolumn{4}{|l|}{ Cuentos de Hadas } \\
\hline \multicolumn{4}{|l|}{$\begin{array}{l}\text { Cuentos populares y } \\
\text { leyendas }\end{array}$} \\
\hline \multicolumn{4}{|l|}{ Histórico } \\
\hline \multicolumn{4}{|l|}{ Biográfico } \\
\hline \multicolumn{4}{|l|}{ Poesía } \\
\hline \multicolumn{4}{|l|}{ Drama } \\
\hline Otros & & & \\
\hline
\end{tabular}

Fig. 7. Géneros y selección de lecturas.

Para que la biblioteca de aula funcione, el docente tiene que incitar a los potenciales sujetos lectores a que lean en un programa prometedor de lectura extensiva. Los lectores tienen que estar deseosos de sumergirse en múltiples tramas de ficción en las cuáles tendrán oportunidades de aprender y de enseñar. Del mismo modo, la retroalimentación que reciben de sus compañeros es uno de los índices más influyentes en la elección del libro. A veces, este intercambio de ideas ocurre tan sólo en el círculo de amistades y no se hace extensible al resto del aula. Si se quiere evitar esta segmentación, el docente puede invitar a algunos de los discentes a compartir sus sensaciones con el resto del grupo-clase. De esta forma nos aseguramos una recepción homogénea (compartida) de la información. En última instancia, serán los alumnos los que elegirán libremente sus lecturas amparados por sus intereses personales y contextuales.

En el otro vértice encontramos la opinión que el docente transmite acerca de un libro. Ésta suele ser muy bien recibida por los estudiantes pero, no obstante, se puede convertir 
en un arma de doble filo ya que pueden llegar a trivializar su percepción y amoldarse a la interpretación emitida por el profesor.

\section{CONCLUSIÓN}

A lo largo de este artículo hemos planteado el dualismo existente a la hora de trabajar la destreza lectora en el aula de ILE. Por un lado nos encontramos con la postura más extendida pero que anula casi en su totalidad la interacción del lector con el texto (lectura intensiva). Lo importante es comprender el texto desde un punto de vista léxico-sintáctico, sin importar si el texto responde o no a los intereses de los sujetos que leen. Por otro lado, hemos hecho una revisión crítica del paradigma de lectura extensiva y cómo se podría articular para su efectividad dentro del aula de idiomas. Si conseguimos que poco a poco el lector dialogue con el texto, incluso cuando éste se encuentre en una lengua extranjera, podremos presenciar cómo la lectura contribuye a la mejora sustancial de su competencia lingüística.

\section{REFERENCIAS BIBLIOGRÁFICAS}

BAmFord, J. y R. R. DAy. 1997. "Extensive reading: What is it? Why bother?". The Language Teacher Online 21, 5: 6-12.

BeLL, T. 1998. "Extensive reading: Why? and How?”. The Internet TESL Journal IV, 12.

Brown, H. D. 2001. Teaching by principles. An interactive approach to language pedagogy. New York: Longman.

DAvis, C.1995. "Extensive reading: an expensive extravagance". ELT Journal 49, 4: $329-336$.

DAY, R. Y J. BAMFORD. 2000. Extensive reading in the second language classroom. Cambridge: Cambridge University Press.

---.----. 2002. “Top ten principles for teaching extensive reading”. Reading in a Foreign Language 14, 2.

ElLEY, W. B. 1991. “Acquiring literacy in a second language: The Effect of Book-Based Programs”. Language Learning 41, 3: 375-411.

Elley, W. B. y F. Mangubhai. 1983. "The impact of reading on second language learning”. Reading Research Quarterly 19, 1: 53-57.

GRABE, W. 1991. "Current developments in second language reading research". TESOL Quarterly 25: 375-406.

Green, J., y R. L. Oxford. 1995. “A closer look at learner strategies, L2 proficiency, and gender”. TESOL Quarterly 29: 261-297.

Hafiz, F. M. y I. Tudor. 1989. "Extensive reading and the development of language skills". ELT Journal 43, 1: 4 -13. 
Hamp-Lyons, E. 1985. "Two approaches to teaching reading: A classroom-based study". Reading in a Foreign Language 3, 1: 363-373.

HedGe, T. 1987. Using readers in language teaching. Londres y Basingstoke: Macmillan Publishers Ltd.

---.--. 2002. Teaching and learning in the language classroom. Oxford: Oxford University Press.

Huang, Xiaohua y M. Van-Naerssen. 1987. "Learning strategies for oral communication". Applied Linguistics 8/3.

Ikumi Hitosugi, C. y R. R. DAy. 2004. "Extensive reading in Japanese". Reading in a Foreign Language 16/1: 20-39.

Keмво, J. 1993. "Reading: Encouraging and maintaining individual extensive reading". English Teaching Forum 31/2: 36-38.

Kern, R. 2000. Literacy and language teaching. Oxford: Oxford University Press.

Krashen, S. D. 1989. The input hypothesis: Issues and implication. Londres: Longman.

---.--. 1993. The power of reading. Insights from the research. Englewood Colorado: Libraries Unlimited, Inc.

Lituanas, P. M; G. M. Jacobs y W.A. Renandya. 1999. “A study of extensive reading with remedial reading students". Language instructional issues in Asian Classrooms. Eds. Y. M. Cheah \& N. S. Moi. Newark, DE: International Development in Asia Committee, International Reading Association. 89-104.

Lunzer, E., y K. GARDNer. 1979. The effective use of reading. Londres: Heinemann Educational Books for The Schools Council.

Maley, A. 1999. "Surviving the 20th century". English Teaching Professional 10: 3-7.

Mason, B. y S. D. Krashen. 1997. "Can we increase the power of reading by adding more output and/or correction". Texas Papers in Foreign Language Education. 20 octubre 2010. $<$ http://www.langconcepts.com/er/maskras.html $>$.

MezynsKi, K. 1983. "Issues concerning the acquisition of knowledge: Effects of vocabulary training on reading comprehension". Review of Educational Research 53: $253-79$.

Nation, P. 2001. Learning vocabulary in another language. Cambridge: Cambridge University Press.

NeLL, V. 1988. Lost in a book: The psychology of reading for pleasure. New Haven, CT: Yale University Press.

NunAn, D. 1991. Language teaching methodology: A textbook for teachers. Londres: Prentice Hall.

PARAN, A. 1996. "Reading in EFL: Facts and fictions". ELT Journal 50, 1: 25 - 34. 
Ruiz Cecilia, R. 2005. Historia corta y lectura extensiva en ILE. El género juvenil de misterio y su recepción entre alumnado de magisterio. Granada: Editorial Universidad de Granada.

SAmuels, S. J. 1991. "Ten best ideas for reading teachers". Ten Best Ideas for Reading Teachers. Ed. E. Fry. Menlo Park: Addison-Wesley. 17 - 20.

Saragi, T.; I.S.P Nation y G.F. Meister. 1978. "Vocabulary learning and reading". System 6, 2: $72-78$.

Stahl, S. A., y C. H. Clark. 1987. "The effects of participatory expectations in classroom discussion on the learning of Science vocabulary". American Educational Research Journal 24, 4: 541-55.

Susser B. y T. N. RobB. 1990. "EFL extensive reading instruction: Research and procedure". JALT Journal 12, 2: $161-185$.

WeLCH, R. A. 1997. "Introducing extensive reading". The Language Teacher Online. 22 octubre 2010. <http://www.jalt-publications.org/tlt/files/97/may/shr_welch. html>. 